\title{
Josephson effect in a multiorbital model for $\mathrm{Sr}_{2} \mathrm{RuO}_{4}$
}

\author{
Kohei Kawai \\ Department of Applied Physics, Nagoya University, Nagoya 464-8603, Japan \\ Keiji Yada and Yukio Tanaka \\ Department of Applied Physics, Nagoya University, Nagoya 464-8603, Japan \\ and Moscow Institute of Physics and Technology, Dolgoprudny, Moscow 141700, Russia \\ Yasuhiro Asano \\ Department of Applied Physics, Hokkaido University, Sapporo 060-8628, Japan \\ and Moscow Institute of Physics and Technology, Dolgoprudny, Moscow 141700, Russia
}

Alexander A. Golubov

Faculty of Science and Technology and MESA+ Institute of Nanotechnology, University of Twente, 7500 AE, Enschede, The Netherlands and Moscow Institute of Physics and Technology, Dolgoprudny, Moscow 141700, Russia

Satoshi Kashiwaya

National Institute of Advanced Industrial Science and Technology (AIST), Tsukuba 305-8568, Japan

and Moscow Institute of Physics and Technology, Dolgoprudny, Moscow 141700, Russia

(Received 24 February 2017; published 24 May 2017; corrected 1 June 2017)

\begin{abstract}
We study Josephson currents between $s$-wave/spin-triplet superconductor junctions by taking into account details of the band structures in $\mathrm{Sr}_{2} \mathrm{RuO}_{4}$, such as three conduction bands and spin-orbit interactions in the bulk and at the interface. We assume five superconducting order parameters in $\mathrm{Sr}_{2} \mathrm{RuO}_{4}$ : a chiral $p$-wave symmetry and four helical $p$-wave symmetries. We calculate the current-phase relationship $I(\varphi)$ in these junctions, where $\varphi$ is the macroscopic phase difference between the two superconductors. The results for a chiral $p$-wave pairing symmetry show that a $\cos (\varphi)$ term appears in the current-phase relation because of time-reversal symmetry (TRS) breaking. On the other hand, this $\cos (\varphi)$ term is absent in the helical pairing states that preserve TRS. We also study the dependence of the maximum Josephson current $I_{c}$ on an external magnetic flux $\Phi$ in a corner junction. The calculated $I_{c}(\Phi)$ obeys $I_{c}(\Phi) \neq I_{c}(-\Phi)$ in a chiral state and $I_{c}(\Phi)=I_{c}(-\Phi)$ in a helical state. We calculate $I_{c}(\Phi)$ in a corner superconducting quantum interference device (SQUID) and a symmetric SQUID geometry. In the latter geometry, $I_{c}(\Phi)=I_{c}(-\Phi)$ is satisfied for all the pairing states and it is impossible to distinguish a chiral state from a helical one. On the other hand, a corner SQUID always gives $I_{c}(\Phi) \neq I_{c}(-\Phi)$ and $I_{c}(\Phi)=I_{c}(-\Phi)$ for a chiral and a helical state, respectively. Experimental tests of these relations in corner junctions and SQUIDs may serve as a tool for unambiguously determining the pairing symmetry in $\mathrm{Sr}_{2} \mathrm{RuO}_{4}$.
\end{abstract}

DOI: 10.1103/PhysRevB.95.174518

\section{INTRODUCTION}

Strontium ruthenate $\left(\mathrm{Sr}_{2} \mathrm{RuO}_{4}\right.$, or $\left.\mathrm{SRO}\right)$ has attracted much interest for its unconventional superconductivity below the critical temperature $T_{c} \sim 1.5 \mathrm{~K}[1]$. The constancy of the Knight shift across $T_{c}$ is strongly indicative of spin-triplet pairing order [2-5]. Many theoretical studies have examined the microscopic mechanism of spin-triplet pairings in this material [6-20]. Exotic phenomena specific to spin-triplet superconductors [21-25] are therefore naturally expected in SRO. Although several studies have focused on the superconducting order parameter, the symmetry of a Cooper pair is not yet fully understood. Five spin-triplet pairing states are compatible with the tetragonal crystal structure of SRO [4]. One of these is a spin-triplet chiral $p$-wave state (denoted the $E_{u}$ state in the Mulliken notation) where the $d$ vector is parallel to $c$ axis of the crystal. The other four candidates are called spin-triplet helical states (denoted $A_{1 u}, A_{2 u}, B_{1 u}$, and $B_{2 u}$ in the Mulliken notation), where the $d$ vectors lie in the $a b$ plane of the crystal.
According to the recently proposed topological classification [26-30], all of the proposed superconducting states are topologically nontrivial. Consequently, topologically protected Andreev bound states are expected at an SRO surface [31]. Some experimental results are consistent with the proposed pair potential. It has been suggested that the maximum Josephson current in $\mathrm{Au}_{0.5} \mathrm{In}_{0.5}$-SRO superconducting quantum interference devices (SQUID) displays an odd-parity pairing state [32].

Tunneling spectroscopy experiments also suggest the formation of a dispersive surface Andreev bound state (SABS) at the in-plane edges of SRO [31,33,34]. The dispersive SABSs $[35,36]$ are distinguishable from the dispersionless SABS in a $d$-wave superconductor. The former generates a broad zerobias conductance peak (ZBCP) [37-39], whereas the latter forms a sharp ZBCP [40-42]. Because SRO is a multiband superconductor, the numerically determined energy dispersion of an SABS in a multiband model is more complicated than that in a single-orbital model $[43,44]$. Yada et al. successfully explained the variety of conductance spectra observed in 
experiments [31] in terms of the three-band degrees of freedom [45]. Several Josephson-junction experiments suggested the presence of domain structures, detected from an anomalous current-switching behavior [46-50]. These experimental findings are consistent with the existence of both chiral and helical $p$-wave pairing symmetries in SRO.

A chiral state is qualitatively different from the four helical states because it breaks the time-reversal symmetry (TRS), whereas the helical states preserve TRS [51]. Although the presence or absence of TRS in SRO is an important issue, experimental results remain controversial. TRS breaking can be verified by observing a spontaneous magnetic field or a spontaneous edge current. Theoretical studies have shown that the amplitude of the spontaneous magnetization is detectable experimentally [52] and that the edge current is robust with respect to surface roughness [53]. Measurements of muon spin resonance and of the Kerr effect have detected the presence of an internal magnetic field [54,55], which in turn suggests a chiral $p$-wave symmetry. On the other hand, scanning SQUID experiments have not shown any signs of a spontaneous magnetic field [56,57], which suggests a helical $p$-wave symmetry. Several theoretical proposals have been put forward to explain the absence of the edge current in SRO [16,58-61]. A resolution of this paradox requires an experimental test able to distinguish unambiguously between a chiral and a helical pairing symmetry.

In this paper, we present a theory of the Josephson effect between a spin-singlet $s$-wave superconductor and a spintriplet $p$-wave superconductor by taking into account the three bands of the SRO in addition to the spin-orbit interaction in the bulk and at the interface. The importance of multiorbital effects are apparent in various physical quantities [14,62]. Since spin-orbit coupling influences the current-phase relation fundamentally, it is necessary that our theory consider a three-band model. We calculated the current-phase relation $I(\varphi)$ in Josephson junctions, where $\varphi$ is the macroscopic phase difference between the two superconductors. We found that $\cos (\varphi)$ appears in $I(\varphi)$ for chiral $p$-wave pairing, owing to TRS breaking, to ensure consistency with previous results [63]. However, $\cos (\varphi)$ is absent for helical pairing, thus reflecting time-reversal invariance. In the case of helical pairing, $\sin (\varphi)$ appears only in a three-band model. We also studied the dependence of the maximum Josephson current $I_{c}$ on an external magnetic flux $\Phi$ in two types of SQUID geometries: a corner SQUID and a symmetric SQUID. In a corner Josephson junction and a corner SQUID, we found $I_{c}(\Phi) \neq I_{c}(-\Phi)$ for a chiral state, whereas $I_{c}(\Phi)=I_{c}(-\Phi)$ holds true for a helical state. We show that the three-band character affects the oscillation period of $I_{c}(\Phi)$. It is possible to determine the pairing symmetry unambiguously by testing these relations in SRO-based corner junctions and SQUIDs. In a symmetric SQUID, the relation $I_{c}(\Phi)=I_{c}(-\Phi)$ is satisfied in both chiral and helical cases.

\section{MODEL AND FORMULATIONS}

This section introduces a model Hamiltonian for an $\mathrm{SRO} /$ normal-metal $(\mathrm{NM}) / s$-wave superconductor junction system. First, we explain the Hamiltonian for bulk SRO, which consists of three terms, $\mathcal{H}_{\text {kin }}, \mathcal{H}_{\text {soi }}$, and $\mathcal{H}_{\text {pair }}$. The first term $\mathcal{H}_{\text {kin }}$ expresses the kinetic energy. Angle-resolved photoemission spectroscopy (ARPES) measurements and firstprinciples calculations have shown that SRO has three twodimensional Fermi surfaces [64-67]. These Fermi surfaces were reproduced by considering three orbitals, i.e., the $d_{x y}$, $d_{y z}$, and $d_{z x}$ orbitals, in SRO. We can therefore consider a three-band two-dimensional Hamiltonian constructed using the tight-binding model:

$$
\mathcal{H}_{\text {kin }}=\sum_{\boldsymbol{k}, \sigma} \hat{c}_{\boldsymbol{k} \sigma}^{\dagger}\left(\begin{array}{ccc}
\varepsilon_{y z}(\boldsymbol{k}) & g(\boldsymbol{k}) & 0 \\
g(\boldsymbol{k}) & \varepsilon_{z x}(\boldsymbol{k}) & 0 \\
0 & 0 & \varepsilon_{x y}(\boldsymbol{k})
\end{array}\right) c_{\boldsymbol{k} \sigma},
$$

where $\boldsymbol{k}$ is a wave number, $\sigma$ is the spin, and $\hat{c}_{\boldsymbol{k} \sigma}=$ $\left(c_{k, \sigma}^{y z}, c_{k, \sigma}^{z x}, c_{k,-\sigma}^{x y}\right)^{T}$ is the annihilation operator. The matrix components of Eq. (1) are given by

$$
\begin{gathered}
\varepsilon_{x y}(\boldsymbol{k})=-2 t_{1}\left(\cos k_{x}+\cos k_{y}\right)-4 t_{2} \cos k_{x} \cos k_{y}-\mu_{x y} \\
\varepsilon_{y z}(\boldsymbol{k})=-2 t_{4} \cos k_{x}-2 t_{3} \cos k_{y}-\mu_{y z} \\
\varepsilon_{z x}(\boldsymbol{k})=-2 t_{3} \cos k_{x}-2 t_{4} \cos k_{y}-\mu_{z x} \\
g(\boldsymbol{k})=-4 t_{5} \sin k_{x} \sin k_{y}
\end{gathered}
$$

where $t_{1}, t_{2}, t_{3}, t_{4}$, and $t_{5}$ are the hopping integrals up to next-nearest-neighbor sites. The second term $\mathcal{H}_{\text {soi }}$ denotes the spin-orbit interaction in bulk SRO,

$$
\mathcal{H}_{\mathrm{soi}}=\lambda \sum_{k, \sigma} \hat{c}_{\boldsymbol{k} \sigma}^{\dagger}\left(\begin{array}{ccc}
0 & i s_{\sigma} & -s_{\sigma} \\
-i s_{\sigma} & 0 & i \\
-s_{\sigma} & -i & 0
\end{array}\right) \hat{c}_{\boldsymbol{k} \sigma},
$$

where $s_{\sigma}=1\left(s_{\sigma}=-1\right)$ for $\sigma=\uparrow(\sigma=\downarrow)$. This term mixes the spin and orbital degrees of freedom. The third term $\mathcal{H}_{\text {pair }}$ expresses the pair potential in SRO. We chose spin-triplet chiral and helical $p$-wave pairings in the following analysis. In the chiral $p$-wave case, we considered a pair potential which belongs to the $E_{u}$ irreducible representation. In the helical $p$-wave case, we considered two kinds of pair potentials belonging to the $A_{u}$ and $B_{u}$ irreducible representations. Using the orbital-dependent $d$ vector $d^{\ell}(\boldsymbol{k})$, the pair potential can be expressed as

$$
\mathcal{H}_{\text {pair }}=\sum_{\ell} \hat{c}^{\ell \dagger}\left(\begin{array}{cc}
\hat{0} & \hat{\Delta}^{\ell}(\boldsymbol{k}) \\
-\hat{\Delta}^{\ell *}(-\boldsymbol{k}) & \hat{0}
\end{array}\right) \hat{c}^{\ell},
$$

with $\hat{c}^{\ell}=\left(c_{\boldsymbol{k}, \uparrow}^{\ell}, c_{\boldsymbol{k}, \downarrow}^{\ell}, c_{-\boldsymbol{k}, \uparrow}^{\ell \dagger}, c_{-\boldsymbol{k}, \downarrow}^{\ell \dagger}\right)^{T}$, and $\hat{\Delta}^{\ell}(\boldsymbol{k})=i \boldsymbol{d}^{\ell}(\boldsymbol{k}) \cdot \boldsymbol{\sigma} \sigma_{y}$, where $\ell$ denotes the orbital index. The five kinds of $d$ vectors are given by

$$
\begin{aligned}
\boldsymbol{d}_{E u}^{y z} & =\hat{z} \Delta_{1}\left(\delta \sin k_{x}+i \sin k_{y}\right), \\
\boldsymbol{d}_{E u}^{z x} & =\hat{z} \Delta_{1}\left(\sin k_{x}+i \delta \sin k_{y}\right), \\
\boldsymbol{d}_{E u}^{x y} & =\hat{z} \Delta_{2}\left(\sin k_{x}+i \sin k_{y}\right), \\
\boldsymbol{d}_{A 1 u}^{y z} & =\hat{x} \delta \Delta_{1} \sin k_{x}+\hat{y} \Delta_{1} \sin k_{y}, \\
\boldsymbol{d}_{A 1 u}^{z x} & =\hat{x} \Delta_{1} \sin k_{x}+\hat{y} \delta \Delta_{1} \sin k_{y}, \\
\boldsymbol{d}_{A 1 u}^{x y} & =\hat{x} \Delta_{2} \sin k_{x}+\hat{y} \Delta_{2} \sin k_{y},
\end{aligned}
$$




$$
\begin{aligned}
& \boldsymbol{d}_{A 2 u}^{y z}=\hat{x} \Delta_{1} \sin k_{y}-\hat{y} \delta \Delta_{1} \sin k_{x}, \\
& \boldsymbol{d}_{A 2 u}^{z x}=\hat{x} \delta \Delta_{1} \sin k_{y}-\hat{y} \Delta_{1} \sin k_{x}, \\
& \boldsymbol{d}_{A 2 u}^{x y}=\hat{x} \Delta_{2} \sin k_{y}-\hat{y} \Delta_{2} \sin k_{x}, \\
& \boldsymbol{d}_{B 1 u}^{y z}=\hat{x} \Delta_{1} \sin k_{x}-\hat{y} \delta \Delta_{1} \sin k_{y}, \\
& \boldsymbol{d}_{B 1 u}^{z x}=\hat{x} \delta \Delta_{1} \sin k_{x}-\hat{y} \Delta_{1} \sin k_{y}, \\
& \boldsymbol{d}_{B 1 u}^{x y}=\hat{x} \Delta_{2} \sin k_{x}-\hat{y} \Delta_{2} \sin k_{y}, \\
& \boldsymbol{d}_{B 2 u}^{y z}=\hat{x} \delta \Delta_{1} \sin k_{y}+\hat{y} \Delta_{1} \sin k_{x}, \\
& \boldsymbol{d}_{B 2 u}^{z x}=\hat{x} \Delta_{1} \sin k_{y}+\hat{y} \delta \Delta_{1} \sin k_{x}, \\
& \boldsymbol{d}_{B 2 u}^{x y}=\hat{x} \Delta_{2} \sin k_{y}+\hat{y} \Delta_{2} \sin k_{x} .
\end{aligned}
$$

In these pair potentials, we only considered the intraorbital pairing cases. Furthermore, we introduced anisotropy in the pair potential in quasi-one-dimensional $d_{y z}$ and $d_{z x}$ orbitals by setting $\delta<1$. In addition, the crystalline symmetry of SRO allows different magnitudes of the pair potential for the twodimensional $d_{y z}$ orbital $\left(\Delta_{1}\right)$ and the quasi-one-dimensional $d_{y z}$ and $d_{z x}$ orbitals $\left(\Delta_{2}\right)$.

In the NM region between an SRO and an $s$-wave superconductor, we considered a single-orbital model given by

$$
\mathcal{H}_{\mathrm{NM}}=\sum_{\boldsymbol{k} \sigma}\left(\varepsilon_{\boldsymbol{k}}-\mu\right) c_{\boldsymbol{k} \sigma}^{\dagger} c_{\boldsymbol{k} \sigma},
$$

where $c_{k \sigma}$ is the annihilation operator for an electron in the NM. The energy dispersion of the NM is given by

$$
\varepsilon_{k}=-2 t_{1}\left[\cos \left(k_{x}\right)+\cos \left(k_{y}\right)\right]-4 t_{2} \cos \left(k_{x}\right) \cos \left(k_{y}\right)-\mu_{n},
$$

where $t$ is the hopping integral between nearest-neighbor sites. We took into account the interface Rashba spin-orbit coupling in the NM layer next to the $\mathrm{SRO}$, which is given by

$$
\mathcal{H}_{\mathrm{RSOI}}=\lambda_{R} \sin k_{y} \hat{\sigma}_{z} .
$$

In the spin-singlet $s$-wave superconductor region, we considered the on-site pair potential as well as the kinetic-energy term in Eq. (13):

$$
\mathcal{H}_{\text {s-wave }}=\sum_{\boldsymbol{k}} \Delta e^{i \varphi} c_{\boldsymbol{k} \uparrow}^{\dagger} c \dagger_{-\boldsymbol{k} \downarrow}+\text { c.c. }
$$

where $\varphi$ is the macroscopic phase of the pair potential relative to the interface normal of the $p$-wave superconductor. These three parts are coupled via hopping at the interface. The magnitude of the hopping at the interface between the NM and the $s$-wave superconductor was chosen to be the same as in the NM. The SRO-NM interface displays three kinds of hopping: $t_{x y}, t_{y z}$, and $t_{z x}$. The first, $t_{x y}$, corresponds to the hopping between the NM and the $d_{x y}$ orbital of SRO. Likewise, $t_{y z}\left(t_{z x}\right)$ also denotes the interface hopping between NM and $d_{y z}\left(d_{z x}\right)$ orbital of SRO.

We calculated the current-phase relation of the Josephson current in the single junction [see Fig. 1(a)] based on a lattice Green's-function method that takes into account the Andreev reflection and Andreev bound states at the interface $[68,69]$. For that purpose, we calculated the Green's function in the superconducting $\mathrm{SRO} / \mathrm{NM} / s$-wave superconductor junction. These three regions are aligned in the (100) direction, with

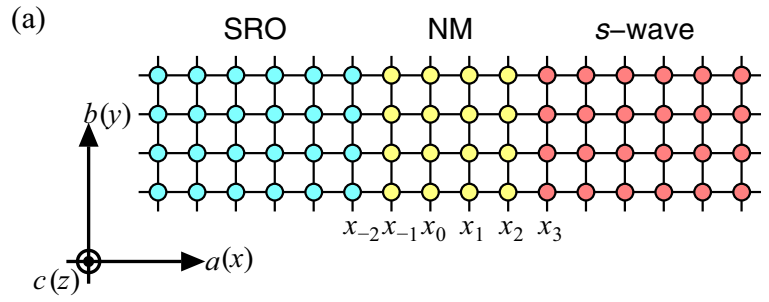

(b)

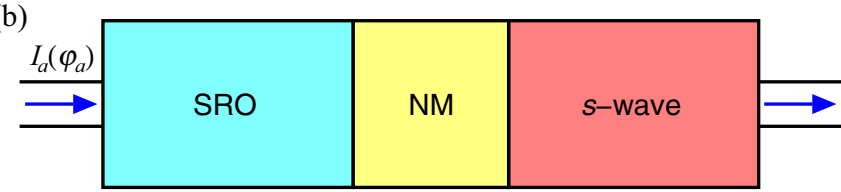

FIG. 1. (a) Lattice model of the junction considered in this paper. (b) Schematic illustrations of an $\mathrm{SRO}\left(\mathrm{Sr}_{2} \mathrm{RuO}_{4}\right) / \mathrm{NM}$ (normal metal) $/ s$-wave superconductor single Josephson junction.

the boundaries for the $s$-wave superconductor and SRO located at $x \leqslant x_{-2}$ and $x \geqslant x_{3}$, respectively. In the numerical calculations, four NM layers are inserted between these two superconductors at $x_{-1} \leqslant x \leqslant x_{2}$. Since we are considering flat interfaces in the ballistic limit, $k_{y}$ is a conserved quantity. In order to obtain the Green's function in this junction, we first calculated the surface Green's functions of the semi-infinite SRO and spin-singlet $s$-wave superconductor, where the surfaces are not coupled to the NM layer. These calculations were based on the recursive Green's function method, using Möbius transformation [70]. Next, we added the two NM layers on these surfaces with the following recursive equation:

$$
\begin{aligned}
& \hat{G}_{n}^{L}\left(k_{y}, i \omega_{l}\right)=\left(i \omega_{l}-\hat{\varepsilon}_{n}\left(k_{y}\right)-\hat{t}_{n, n-1} \hat{G}_{n-1}^{L}\left(k_{y}, i \omega_{l}\right) \hat{t}_{n-1, n}\right)^{-1}, \\
& \hat{G}_{n}^{R}\left(k_{y}, i \omega_{l}\right)=\left(i \omega_{l}-\hat{\varepsilon}_{n}\left(k_{y}\right)-\hat{t}_{n, n+1} \hat{G}_{n+1}^{L}\left(k_{y}, i \omega_{l}\right) \hat{t}_{n+1, n}\right)^{-1},
\end{aligned}
$$

where $G_{n}^{L I}\left(k_{y}, i \omega_{l}\right)$ stands for the surface Green's function for the system on the left (right) side of the interface, with $x \leqslant x_{n}$ $\left(x \geqslant x_{n}\right)$. The operators $\hat{\varepsilon}_{n}\left(k_{y}\right)$ and $\hat{t}_{n, n-1}$ represent the local and nonlocal parts of the Hamiltonian. Then, we obtained two surface Green's functions, defined for $x \leqslant x_{0}$ and $x \geqslant x_{1}$. These two systems are combined in the equations

$$
\begin{aligned}
& \hat{G}_{00}\left(k_{y}, i \omega_{l}\right)=\left\{\left[\hat{G}_{0}^{L}\left(k_{y}, i \omega_{l}\right)\right]^{-1}-\hat{t}_{01} \hat{G}_{1}^{R}\left(k_{y}, i \omega_{l}\right) \hat{t}_{10}\right\}^{-1}, \\
& \hat{G}_{11}\left(k_{y}, i \omega_{l}\right)=\left\{\left[\hat{G}_{1}^{R}\left(k_{y}, i \omega_{l}\right)\right]^{-1}-\hat{t}_{10} \hat{G}_{0}^{L}\left(k_{y}, i \omega_{l}\right) \hat{t}_{01}\right\}^{-1} .
\end{aligned}
$$

Then, we obtained the nonlocal Green's functions in the $s$-wave/NM/SRO junction as follows:

$$
\begin{aligned}
& \hat{G}_{01}\left(k_{y}, i \omega_{l}\right)=\hat{G}_{0}^{L}\left(k_{y}, i \omega_{l}\right) \hat{t}_{01} \hat{G}_{11}\left(k_{y}, i \omega_{l}\right), \\
& \hat{G}_{10}\left(k_{y}, i \omega_{l}\right)=\hat{G}_{1}^{R}\left(k_{y}, i \omega_{l}\right) \hat{t}_{10} \hat{G}_{00}\left(k_{y}, i \omega_{l}\right) .
\end{aligned}
$$

The Fourier transforms of $\hat{G}_{01}\left(k_{y}, i \omega_{l}\right)$ and $\hat{G}_{10}\left(k_{y}, i \omega_{l}\right)$ are given by

$$
\hat{G}_{01}\left(k_{y}, \tau\right)=\frac{1}{\beta} \sum_{l} \hat{G}_{01}\left(k_{y}, i \omega_{l}\right) e^{-i \omega_{l} \tau},
$$




$$
\hat{G}_{10}\left(k_{y}, \tau\right)=\frac{1}{\beta} \sum_{l} \hat{G}_{10}\left(k_{y}, i \omega_{l}\right) e^{-i \omega_{l} \tau},
$$

with $\beta=1 /\left(k_{B} T\right)$ and where $T$ is the temperature. The above formulas for $\hat{G}_{01}\left(k_{y}, \tau\right)$ and $\hat{G}_{10}\left(k_{y}, \tau\right)$ can be expressed as

$$
\begin{aligned}
& \hat{G}_{01}\left(k_{y}, \tau\right)=-\left\langle T_{\tau}\left[\hat{C}_{0}(\tau) \hat{C}_{1}^{\dagger}\right]\right\rangle, \\
& \hat{G}_{10}\left(k_{y}, \tau\right)=-\left\langle T_{\tau}\left[\hat{C}_{1}(\tau) \hat{C}_{0}^{\dagger}\right]\right\rangle,
\end{aligned}
$$

with

$$
\begin{aligned}
& \hat{C}_{0}^{\dagger}=\left(\begin{array}{llll}
C_{0 e \uparrow}^{\dagger} & C_{0 e \downarrow}^{\dagger} & C_{0 h \uparrow}^{\dagger} & C_{0 h \downarrow}^{\dagger}
\end{array}\right), \\
& \hat{C}_{1}^{\dagger}=\left(\begin{array}{llll}
C_{1 e \uparrow}^{\dagger} & C_{1 e \downarrow}^{\dagger} & C_{1 h \uparrow}^{\dagger} & C_{1 h \downarrow}^{\dagger}
\end{array}\right) .
\end{aligned}
$$

Thus, we obtained the current-phase relation $I(\varphi)$ by using these $\hat{G}_{01}\left(k_{y}, \tau\right)$ and $\hat{G}_{10}\left(k_{y}, \tau\right)$ :

$$
\begin{aligned}
I(\varphi)= & \frac{i e t}{\hbar} \int_{-\pi}^{\pi} \operatorname{Tr}^{\prime}\left[\hat{G}_{01}\left(k_{y}, \tau=-0, \varphi\right)\right. \\
& \left.-\hat{G}_{10}\left(k_{y}, \tau=-0, \varphi\right)\right] d k_{y} \\
= & \frac{i e t}{\hbar} \int_{-\pi}^{\pi} \operatorname{Tr}^{\prime} \frac{1}{\beta} \sum_{l}\left[\hat{G}_{01}\left(k_{y}, i \omega_{l}, \varphi\right)\right. \\
& \left.-\hat{G}_{10}\left(k_{y}, i \omega_{l}, \varphi\right)\right] d k_{y},
\end{aligned}
$$

where $\operatorname{Tr}^{\prime}$ is a partial sum of the diagonal elements of the Hamiltonian, including only those matrix elements that refer to the electron space.

Below, we define the model parameters that were used in the calculations. For the hopping parameters in SRO, we assumed $t_{2} / t_{1}=0.395, t_{3} / t_{1}=1.25, t_{4} / t_{1}=0.125$, and $t_{5} / t_{1}=0.15$, based on first-principles calculations. Here, $t_{1}$ is the nearestneighbor hopping parameter in the $d_{x y}$ orbital in SRO, which first-principles calculations estimate as being approximately $230 \mathrm{meV}$ [4]. Furthermore, the chemical potentials in each orbital in SRO, $\mu_{y z}, \mu_{z x}$, and $\mu_{x y}$, were chosen to yield the following numbers of electron: $n_{y z}=n_{z x}=n_{x y}=2 / 3$. The chemical potential in the normal metal, $\mu_{n}$, was chosen so that the number of electron is $2 / 3$. The magnitude of the spin-orbit interaction in the bulk SRO, expressed as $\lambda$, changes these values. We set $\lambda=0.3$ for consistency with quasiparticle spectra obtained by angle-resolved photoemission spectroscopy [4]. We chose the magnitudes of the pair potential for the $d_{y z}$ and $d_{z x}$ orbitals in SRO to exceed that of the $d_{x y}$ orbital, as determined previously by tunneling spectroscopy $[31,45]$. The magnitude of the pair potential in the $d_{y z}$ and $d_{z x}$ orbitals was set to $\Delta_{1}=0.001 t_{1}$. We set the magnitude of the pair potential for the $d_{x y}$ orbital to $\Delta_{2}=0.4 \Delta_{1}$. For the quasi-one-dimensional nature of the pair potential for the $d_{x y}$ orbital, we set $\delta=0.1$, based on the ratio of $t_{3}$ to $t_{4}$.

We assumed that an $s$-wave superconductor and an NM are described by the same single-orbital model as that of the $d_{x y}$ orbital in SRO. We set their chemical potentials $\mu_{n}$ to the same level as the $d_{x y}$ orbital in SRO, in the absence of spin-orbit interaction in the bulk SRO. The magnitude of the pair potential of the $s$-wave superconductor was set to $\Delta_{s}=10 \Delta_{1}$. The magnitude of the Rashba spin-orbit interaction at the interface between NM and SRO, $\lambda_{R}$, depends on the microscopic
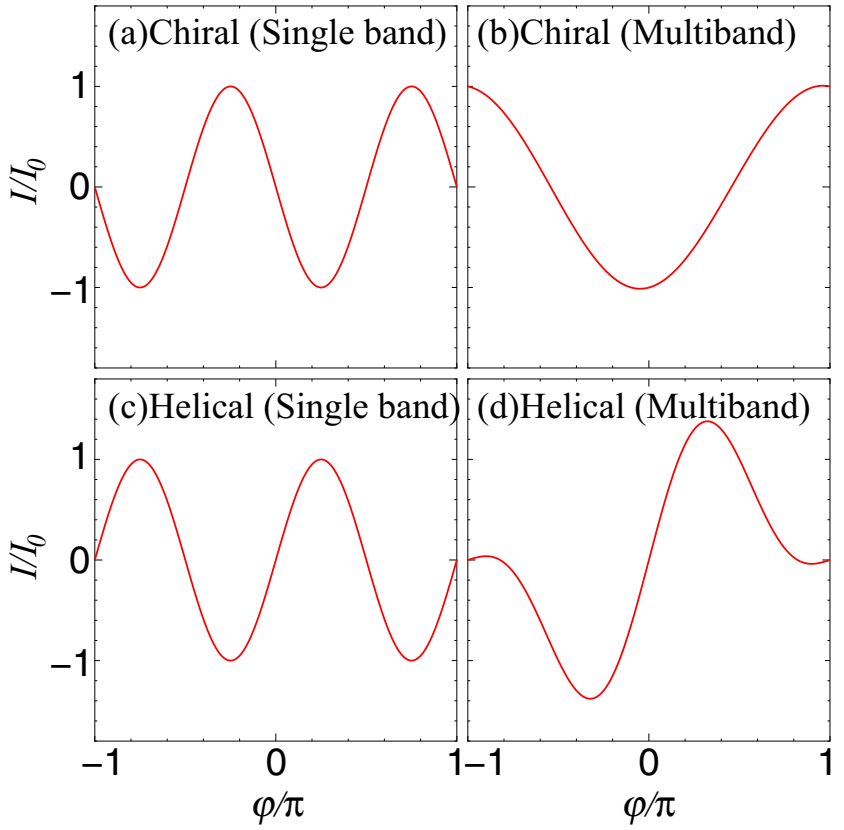

FIG. 2. Current-phase relation in the absence of interface Rashba spin-orbit interaction $\left(\lambda_{R}\right)$ for (a) the chiral $p$ wave $\left(E_{u}\right)$ in the singleband model, (b) the chiral $p$ wave $\left(E_{u}\right)$ in the multiband model, (c) the helical $p$ wave $\left(A_{1 u}\right)$ in the single-band model, and (d) the helical $p$ wave $\left(A_{1 u}\right)$ in the multiband model.

electronic properties of the junction and was set to 0.3 in this study.

\section{RESULTS}

\section{A. Current phase relation}

Figure 2 shows the current-phase relation in the absence of interface Rashba spin-orbit interaction. Here, the Josephson current $I(\varphi)$ is decomposed into the Fourier series

$$
I(\varphi)=\sum_{n=1}^{\infty} I_{n}^{s} \sin (n \varphi)+I_{n}^{c} \cos (n \varphi) .
$$

It is then normalized by $I_{0}$, the maximum value of the Fourier coefficients. Table I shows which of the Fourier coefficients have nonzero values.

As shown in Figs. 2(a) and 2(c), the Josephson current is almost proportional to $\sin (2 \varphi)$ in the case where the first-order Josephson coupling is absent. In fact, Table I shows that only the sinusoidal terms with an even-number order are nonzero. On the other hand, odd-order terms are nonzero in the case of

TABLE I. Fourier series of current-phase relation in the absence of interface Rashba spin-orbit interaction. $\sqrt{ }(-)$ denotes coefficients with a nonzero (zero) value.

\begin{tabular}{lcccc}
\hline \hline & $I_{1}^{s}$ & $I_{1}^{c}$ & $I_{2}^{s}$ & $I_{2}^{c}$ \\
\hline (a) Chiral (single band) & - & - & $\sqrt{ }$ & - \\
(b) Chiral (multiband) & $\sqrt{ }$ & $\sqrt{ }$ & $\sqrt{ }$ & $\sqrt{ }$ \\
(c) Helical (single band) & - & - & $\sqrt{ }$ & - \\
(d) Helical (multiband) & $\sqrt{ }$ & - & $\sqrt{ }$ & - \\
\hline \hline
\end{tabular}



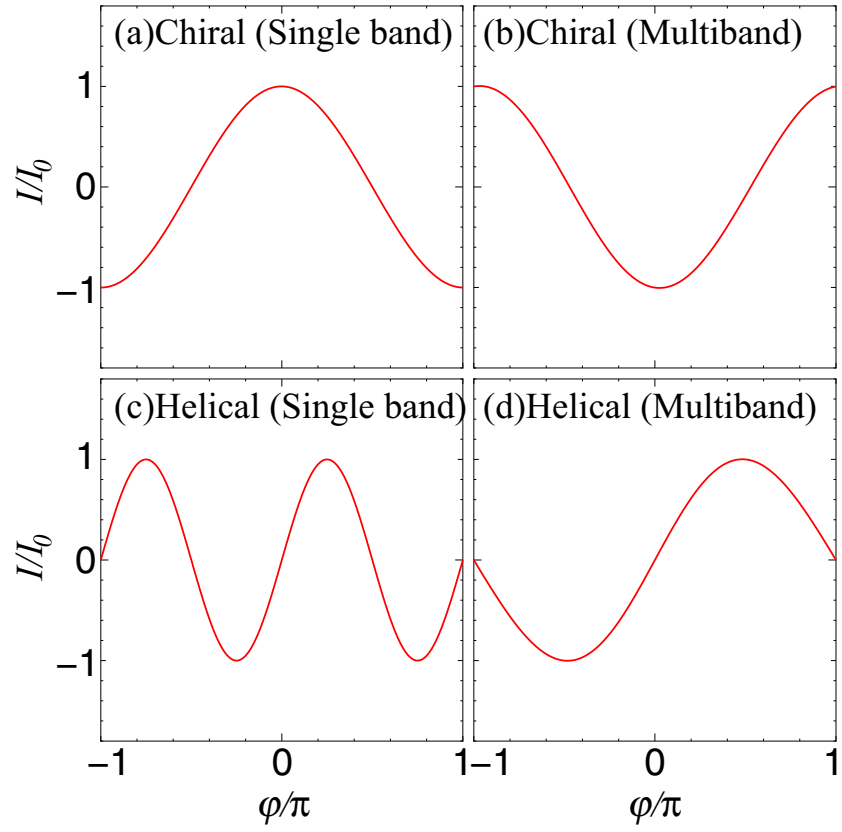

FIG. 3. Current-phase relation $I(\varphi)$ in the presence of interface Rashba spin-orbit interaction $\left(\lambda_{R}>0\right)$ for (a) the chiral $p$ wave $\left(E_{u}\right)$ in the single-band model, (b) the chiral $p$ wave $\left(E_{u}\right)$ in the multiband model, (c) the helical $p$ wave $\left(A_{1 u}\right)$ in the single-band model, and (d) the helical $p$ wave $\left(A_{1 u}\right)$ in the multiband model.

the multiband model, as shown in Fig. 2 and Table I [(b) and (d)]. We confirmed that these odd-order terms are zero in the absence of spin-orbit interaction ( $L S$ coupling) in bulk SRO. We note that the cosine terms appear in the chiral $p$-wave case but are absent in the helical $p$-wave case. The cosine terms in the chiral $p$-wave case are nonzero even in the absence of Rashba spin-orbit coupling $\lambda_{R}$. This is because the hopping integral $t_{5}$ (i.e., corresponding to interorbital hopping between the $d_{y} z$ and $d_{x} z$ orbitals) is nonzero and spin-orbit coupling in bulk SRO $\lambda$ enhances the magnitude of the cosine terms. When the opposite chirality of the pair potential is chosen with

$$
\begin{aligned}
& \boldsymbol{d}_{E u}^{y z}=\hat{z} \Delta_{1}\left(\delta \sin k_{x}-i \sin k_{y}\right), \\
& \boldsymbol{d}_{E u}^{z x}=\hat{z} \Delta_{1}\left(\sin k_{x}-i \delta \sin k_{y}\right), \\
& \boldsymbol{d}_{E u}^{x y}=\hat{z} \Delta_{2}\left(\sin k_{x}-i \sin k_{y}\right),
\end{aligned}
$$

the signs of $I_{1}^{c}$ and $I_{2}^{c}$ are reversed.

We plot the current-phase relations in the presence of interface Rashba spin-orbit coupling $\left(\lambda_{R}>0\right)$ in Fig. 3. Figure 3(c) shows no qualitative difference between the Josephson currents in the presence or absence of interface Rashba spin-orbit interaction, in the single-band model, and in the case of helical pairing. On the other hand, cosine terms appear as a result of the interface Rashba spin-orbit coupling in the case of the chiral $p$-wave shown in Fig. 3(a) [63]. By contrast, there is no qualitative difference between the current-phase relations in the presence or absence of interface Rashba spin-orbit interaction in the multiband model, as shown in Figs. 3(b) and 3(d) and Table II [(b) and (d)]. In the most general case, where both the interface Rashba spin-orbit interaction and bulk $L S$ coupling in the multiband model exist, we observe a qualitative difference between the chiral and
TABLE II. Fourier series of the current-phase relation in the presence of interface Rashba spin-orbit interaction.

\begin{tabular}{lcccc}
\hline \hline & $I_{1}^{s}$ & $I_{1}^{c}$ & $I_{2}^{s}$ & $I_{2}^{c}$ \\
\hline (a) Chiral (single band) & - & $\sqrt{ }$ & $\sqrt{ }$ & - \\
(b) Chiral (multiband) & $\sqrt{ }$ & $\sqrt{ }$ & $\sqrt{ }$ & $\sqrt{ }$ \\
(c) Helical (single band) & - & - & $\sqrt{ }$ & - \\
(d) Helical (multiband) & $\sqrt{ }$ & - & $\sqrt{ }$ & - \\
\hline \hline
\end{tabular}

helical $p$-wave cases. The cosine terms $I_{1}^{c}$ and $I_{2}^{c}$ appear only in the case of chiral $p$-wave pairing. This difference is due to the broken TRS that occurs in chiral $p$-wave pairing. In the following calculations for various junctions, we considered the interface Rashba spin-orbit interactions and used the multiband model.

In order to take into account the corner structure of the junction, we show the relation between the current phase relations in different orientations in Table III. The orientation dependence affects the maximum Josephson current in a corner junction or SQUID when it is written as a function of the external magnetic flux $\Phi$. Although the calculation of the $\Phi$ dependence will be shown in next subsection, we first show the relation between $I_{a}\left(\varphi_{a}\right)$ and $I_{\bar{b}}\left(\varphi_{\bar{b}}\right)$ indicated in Fig. 4. This relation depends on the pairing symmetries specified in Table III. This relation in chiral $p$-wave pairing is different from that in helical $p$-wave pairing. Furthermore, in the helical $p$-wave cases, the relation between $I_{a}\left(\varphi_{a}\right)$ and $I_{\bar{b}}\left(\varphi_{\bar{b}}\right)$ depends on the irreducible representations of the pair potentials. This affects the properties of the corner junction or corner SQUID, as shown in the next subsection. Next, we show the relation between the $I_{a}\left(\varphi_{a}\right)$ and $I_{\bar{a}}\left(\varphi_{\bar{a}}\right)$ indicated in Fig. 1. The equation $I_{a}(\varphi)=I_{\bar{a}}(\varphi+\pi)$ is valid for all pairings. This fact influences the properties of a symmetric SQUID.

\section{B. Magnetic-field dependence of the maximum Josephson current in various junctions}

In this subsection, we calculate the magnetic-field dependence of the maximum Josephson current in corner junctions, corner SQUIDs, and symmetric SQUIDs.

We calculated the relation between the external magnetic flux $\Phi$ and the maximum Josephson current $I_{c}$ by a standard method. In the Josephson junctions shown in Figs. 1 and 4, we assumed that the external magnetic field was applied parallel to the $z$ axis. The vector potential is then given by

$$
\boldsymbol{A}=A_{y}(x) \boldsymbol{y} .
$$

On the other hand, the phase $\gamma$ of the pair potential obeys

$$
\nabla \gamma=\frac{m^{*} \boldsymbol{v}_{s}}{\hbar}+\frac{2 \pi}{\Phi_{0}} \boldsymbol{A}
$$

TABLE III. Relations between $I_{a}\left(\varphi_{a}\right)$ and $I_{\bar{b}}\left(\varphi_{\bar{b}}\right)$ shown in Fig. 1 for chiral $\left(E_{u}\right)$, helical $\left(A_{1 u}, B_{2 u}\right)$, and helical $\left(A_{2 u}, B_{1 u}\right)$ pairings.

\begin{tabular}{lc}
\hline \hline Type of pairing & Relation between $I_{a}\left(\varphi_{a}\right)$ and $I_{\bar{b}}\left(\varphi_{\bar{b}}\right)$ \\
\hline Chiral $\left(E_{u}\right)$ & $I_{a}\left(\varphi_{a}\right)=-I_{\bar{b}}\left(-\varphi_{\bar{b}}+\pi / 2\right)$ \\
Helical $\left(A_{1 u}, B_{2 u}\right)$ & $I_{a}\left(\varphi_{a}\right)=I_{\bar{b}}\left(\varphi_{\bar{b}}\right)$ \\
Helical $\left(A_{2 u}, B_{1 u}\right)$ & $I_{a}\left(\varphi_{a}\right)=I_{\bar{b}}\left(\varphi_{\bar{b}}+\pi\right)$ \\
\hline \hline
\end{tabular}


(a)

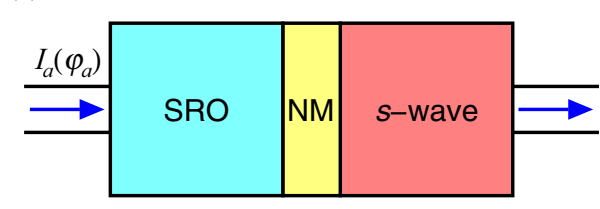

(c)

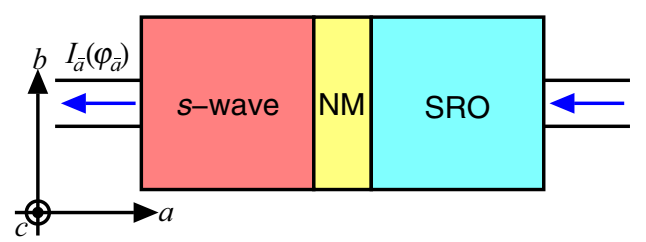

(b)

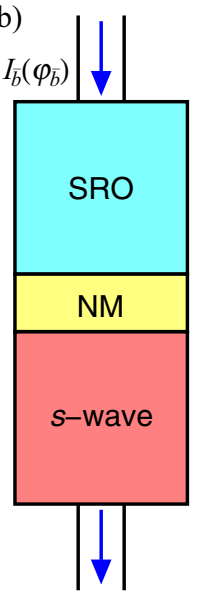

FIG. 4. Schematic illustrations of the SRO $/ \mathrm{NM} / s$-wave superconductor single Josephson junctions considered in this paper. Current-phase relations in junctions (a)-(c) were calculated independently. The results were then combined to calculate the magnetic-field dependence of the corner junction, corner SQUID, and symmetric SQUID.

Since the magnetic field is screened inside the superconductor because of the Meissner effect, $A_{y}(x)$ takes the constant value $A_{y}(\infty)$ found at locations far from the interface. Using these properties, we integrated both sides of the $y$ component of Eq. (32) with respect to $y$,

$$
\gamma(y)=\gamma(0)+\frac{2 \pi}{\Phi_{0}} A(\infty) y .
$$

The phase difference between the $s$-wave superconductor and the SRO is therefore given by

$$
\varphi(y)=\varphi(0)+\frac{2 \pi}{\Phi_{0}}\left[A_{2}(\infty)-A_{1}(\infty)\right] y .
$$

Here, $A_{1}$ and $A_{2}$ represent the vector potentials far from the interface in the SRO and $s$-wave superconductor, respectively. The Fourier components of the Josephson current, $I_{n}^{s}$ and $I_{n}^{c}$, defined in Eq. (29), were obtained in the previous subsection in the absence of a magnetic field. In the presence of a magnetic

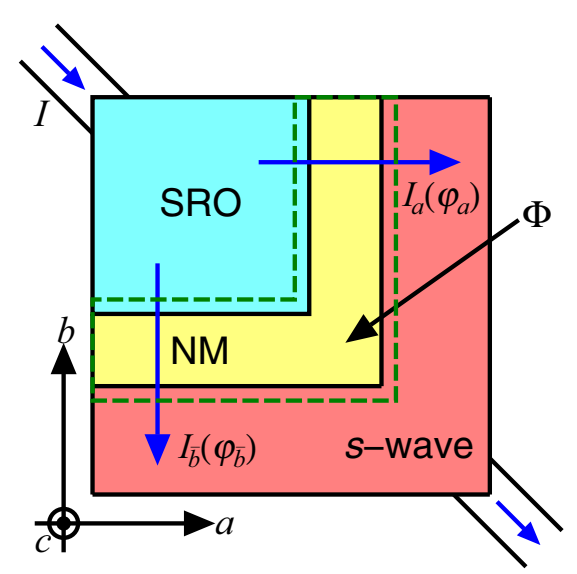

FIG. 5. Schematic illustration of an $\mathrm{SRO} / \mathrm{NM} / s$-wave corner junction.

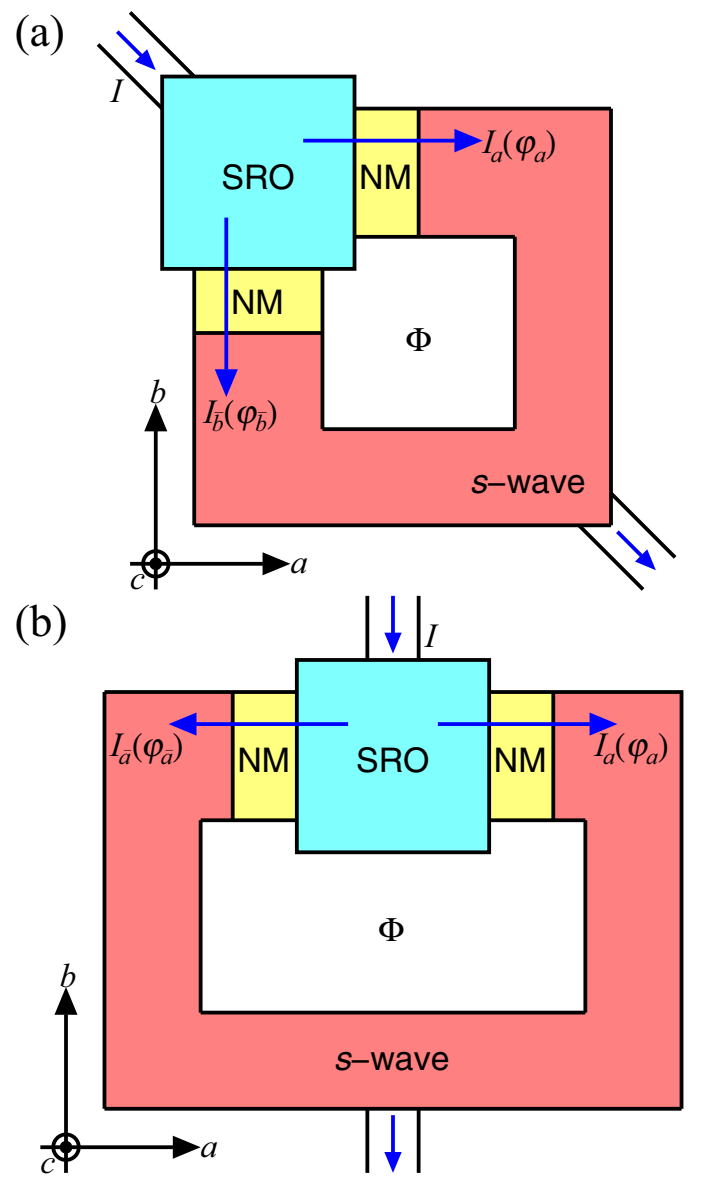

FIG. 6. Schematic illustration of $\mathrm{SRO} / \mathrm{NM} / s$-wave SQUIDs: (a) corner SQUID and (b) symmetric SQUID.

field, the Josephson current becomes a function of $y$. We integrated this function with respect to $y$ :

$$
\begin{aligned}
I(\Phi, \varphi(0))= & Z \int_{-Y / 2}^{Y / 2} I(y) d y \\
= & Y Z \sum_{n=1}^{\infty}\left\{\frac { \operatorname { s i n } ( n \pi \Phi / \Phi _ { 0 } ) } { n \pi \Phi / \Phi _ { 0 } } \left[I_{n}^{s} \sin (n \varphi(0))\right.\right. \\
& \left.\left.+I_{n}^{c} \cos (n \varphi(0))\right]\right\},
\end{aligned}
$$

where, $Y$ and $Z$ are the sizes of the junction. It is evident that Eq. (35) displays a periodicity of $2 \pi$ with respect to $\varphi(0)$. Therefore, by changing $\varphi(0)$ over the range $-\pi \leqq \varphi(0) \leqq$ $\pi$, the maximum Josephson current $I_{c}$ can be obtained as a function of the external magnetic flux $\Phi$.

Next, we calculated the maximum Josephson current $I_{c}$ in the corner junction shown in Fig. 5 as a function of $\Phi$, using a similar approach to that described in [71]. We obtained the current-phase relations $I_{a}\left(\varphi_{a}\right)$ and $I_{\bar{b}}\left(\varphi_{\bar{b}}\right)$ indicated in Fig. 5. By calculating the following equation instead of Eq. (35), we obtained the maximum Josephson current $I_{c}$ as a function of the external magnetic flux $\Phi$ based on $I(\Phi, \varphi(0))$, given by

$$
I(\Phi, \varphi(0))=Z\left[\int_{0}^{Y / 2} I_{a}(y) d y+\int_{-Y / 2}^{0} I_{\bar{b}}(y) d y\right] .
$$



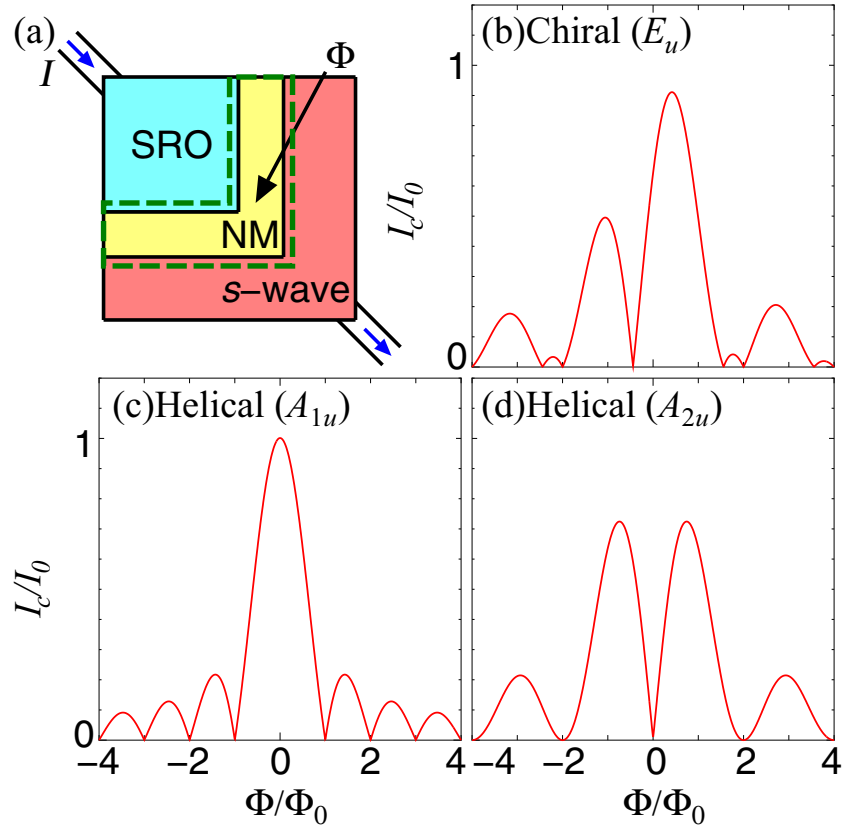

FIG. 7. Fraunhofer pattern in the SRO/NM/s wave. (a) Schematic illustration of a corner junction, and the corresponding Fraunhofer pattern for (b) chiral $\left(E_{u}\right)$ pairing, (c) helical $\left(A_{1 u}\right)$ pairing, and (d) helical $\left(A_{2 u}\right)$ pairing.

Finally, we calculated the maximum Josephson current $I_{c}$ as a function of the external magnetic flux $\Phi$ in the two types of SQUIDs shown in Fig. 6. The macroscopic phase differences of the two superconductors $\varphi_{a}$ and $\varphi_{\bar{b}}$ obey the following relation:

$$
\varphi_{\bar{b}}-\varphi_{a}=\frac{2 \pi \Phi}{\Phi_{0}} .
$$

The total current in these parallel circuits is therefore given by

$$
I(\Phi, \varphi)=I_{a}(\varphi)+I_{\bar{b}}\left(\varphi+\frac{2 \pi \Phi}{\Phi_{0}}\right) .
$$

By evaluating the maximum value of Eq. (37) for a given external magnetic flux $\Phi$, we obtained the maximum Josephson current as a function of $\Phi$.

The $I_{c}$ functions for the corner junction of SRO are plotted in Fig. 7. In the cases of the helical $p$ wave, the positions of the minima depend on the $d$ vector as shown in Figs. 5(b) and 5(c). This is because the relation between the Josephson currents $I_{a}\left(\varphi_{a}\right)$ and $I_{\bar{b}}\left(\varphi_{\bar{b}}\right)$ in Fig. 7 is different for each pairing symmetry. In particular, $I_{a}(\varphi)=I_{b}(\varphi)$ for the $A_{1 u}$ and $B_{2 u}$ pairings, while $I_{a}(\varphi)=I_{b}(\varphi+\pi)$ for the $A_{2 u}$ and $B_{1 u}$ pairings. For all the helical $p$-wave cases, the Fraunhofer patterns are symmetric functions of $\Phi$. On the other hand, $I(\Phi)$ is not a symmetric function of $\Phi$ for chiral $p$-wave pairing. This difference results from the existence of the cosine terms in the current-phase relation. In other words, the broken TRS causes the asymmetry of $I_{c}=I_{c}(\Phi)$, i.e., $I_{c}(\Phi) \neq I_{c}(-\Phi)$. These results are summarized in Table IV. As seen from this table, there are qualitative differences between the helical and chiral $p$-wave pairings. The asymmetry of the Josephson current is due to the existence of cosine terms in the current-phase relation for the chiral $p$-wave pairings. These cosine terms can
TABLE IV. $\Phi$ dependence and zero points of $I(\Phi)$ in an $\mathrm{SRO} / \mathrm{NM} / s$-wave corner junction for (b) chiral $\left(E_{u}\right),(\mathrm{c})$ helical $\left(A_{1 u}\right.$, $\left.B_{2 u}\right)$, and (d) helical $\left(A_{2 u}, B_{1 u}\right)$ pairings in SRO. Schematic illustration of corner junction (a).

\begin{tabular}{lcc}
\hline \hline Type of pairing & $\Phi$ dependence & Zero points of $I(\Phi)$ \\
\hline (a) Chiral $\left(E_{u}\right)$ & asymmetric & $\pm 2 \Phi_{0}, \pm 4 \Phi_{0}, \ldots$ \\
(b) Helical $\left(A_{1 u}, B_{2 u}\right)$ & symmetric & $\pm \Phi_{0}, \pm 2 \Phi_{0}, \ldots$ \\
(c) Helical $\left(A_{2 u}, B_{1 u}\right)$ & symmetric & $\pm 2 \Phi_{0}, \pm 4 \Phi_{0}, \ldots$ \\
\hline \hline
\end{tabular}

be nonzero unless both $\lambda$ and $\lambda_{R}$ are nonzero owing to the presence of interorbital hopping in the multiband model. The magnitudes of these cosine terms and the resulting asymmetry of $I(\Phi)$ are enhanced by the spin-orbit interactions, expressed through $\lambda$ and $\lambda_{R}$.

Next, we discuss $I_{c}$ in the corner SQUID shown in Fig. 8. This $I_{c}$ is symmetric or asymmetric with respect to $\Phi$ for the helical and chiral cases, respectively. As in the case of the SRO/NM/ $s$-wave corner junction, the existence of the cosine terms in the current-phase relation in chiral pairing causes the asymmetry of $I_{c}(\Phi)$. The chiral pairing is consistent with a previous study based on a single-band model [72]. In the cases of helical pairing, the position of the maximum or minimum in $I_{c}(\Phi)$ depends on the pairing symmetry (irreducible representation), i.e., the $d$-vector as shown in Figs. 8(b) and 8(c) (see Table V). We note that the $\Phi_{0}$ periodicity in the helical pairing case appears only for a three-band model.

Finally, we consider the case of the so-called symmetric SQUID [73]. Figure 9 shows the $\Phi$ dependence of $I_{c}$ in the symmetric SQUID shown in Fig. 6(b). In this junction, there is no qualitative difference between the cases of chiral and helical pairing since $I_{a}(\varphi)=I_{\bar{b}}(\varphi+\pi)$ is satisfied. The

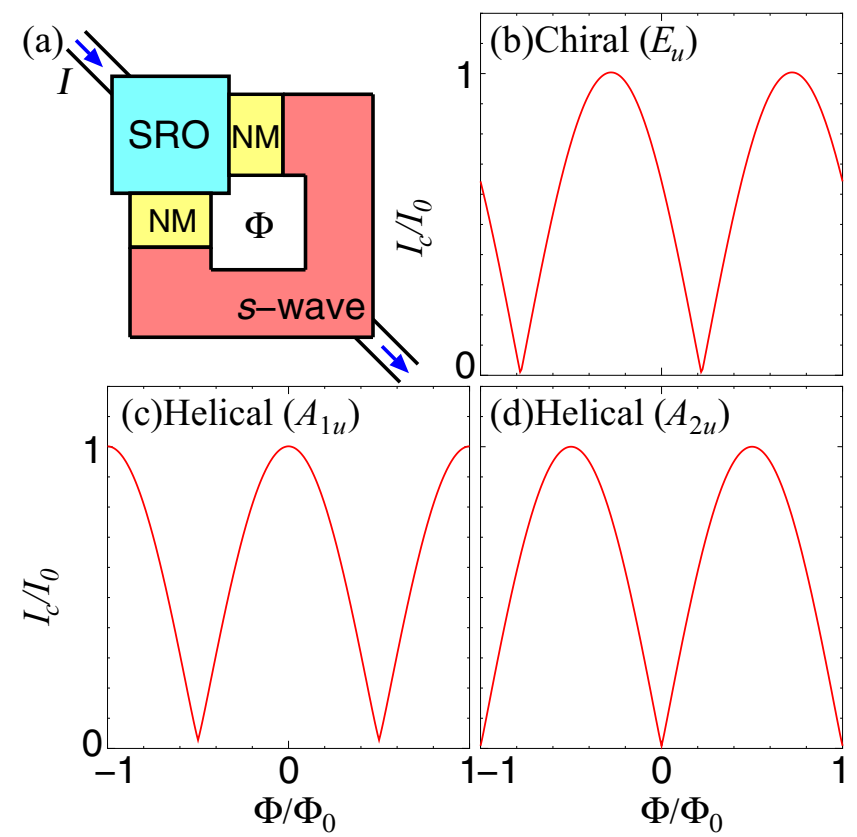

FIG. 8. (a) Maximum Josephson current $I_{c}$ in a corner SQUID for (b) chiral $\left(E_{u}\right)$, (c) helical $\left(A_{1 u}\right)$, and (d) helical $\left(A_{2 u}\right)$ pairings. 
TABLE V. $\Phi$ dependence and period of the maximum Josephson current $I_{c}$ in a corner SQUID.

\begin{tabular}{lcc}
\hline \hline Type of pairing & $\Phi$ dependence & Period \\
\hline (a) Chiral & asymmetric & $\Phi_{0}$ \\
(b), (c) Helical & symmetric & $\Phi_{0}$ \\
\hline \hline
\end{tabular}

resulting Josephson current $I_{c}$ is symmetric for both chiral and helical pairings, including in the presence of the cosine terms. Thus, we do not find any qualitative difference in $I_{c}$ for the chiral and helical pairings in this symmetric SQUID (see Table VI).

\section{DISCUSSION AND SUMMARY}

Here, we discuss the multiband effect on the Josephson current in the present calculations, starting with the chiral $p$-wave case. As shown in Tables I and II, the spin-orbit interaction in the bulk SRO $(\lambda)$ and the interface Rashba spin-orbit interaction $\left(\lambda_{R}\right)$ generate $I_{1}^{s}$ for chiral $p$-wave pairing. We found that the coefficient of the $\sin (\phi)$ term $I_{1}^{S}$ has the form

$$
I_{1}^{s}=\alpha \lambda+\beta t_{5} \lambda_{R}+O\left(\lambda^{2}\right)+O\left(t_{5}^{2} \lambda_{R}^{2}\right),
$$

which is confirmed by Fig. 10. This form suggests that $\lambda$ directly induces $I_{1}^{s}$, whereas the existence of interorbital hopping $t_{5}$ is needed to produce $I_{1}^{s}$ from $\lambda_{R}$. In the single-band model, $I_{1}^{S}$ is absent while $I_{1}^{c}$ is induced by $\lambda_{R}$ in chiral $p$-wave pairing. In the multiorbital model, $t_{5}$ induces the effective phase shift of the pair potential. A part of $I_{1}^{c}$ is then converted to $I_{1}^{s}$ by $t_{5}$. Thus, we can conclude that the existence of $I_{1}^{S}$ results from the multiband model in SRO. This term becomes dominant in the limit of low transmissivities, where the higher-order Josephson couplings are strongly suppressed.

(a)
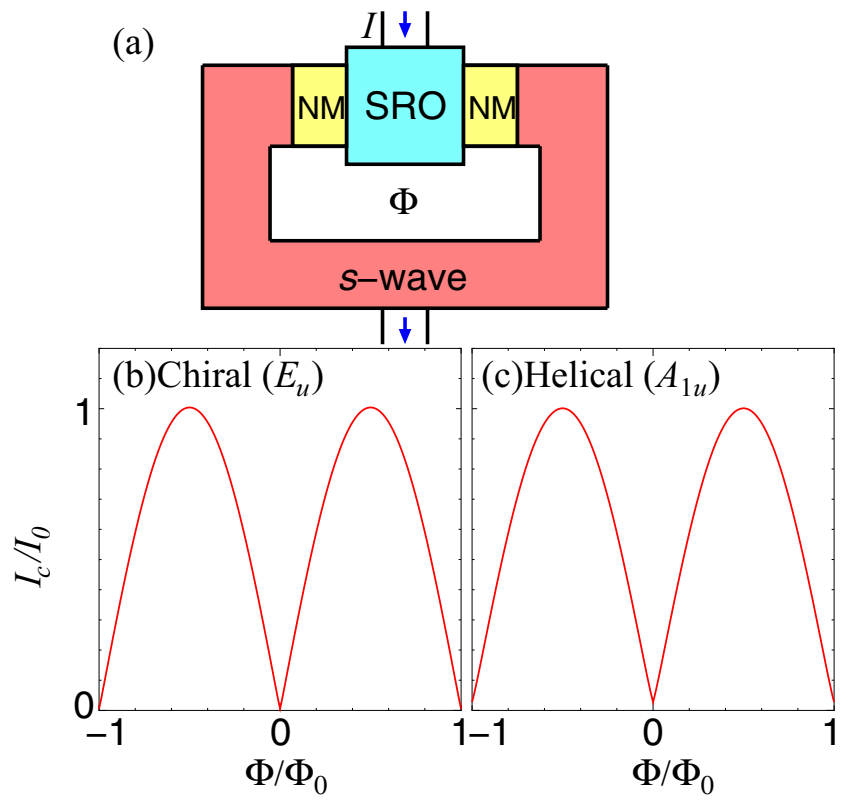

FIG. 9. (a) Symmetric SQUID and the corresponding $I_{c}$ for (b) chiral $\left(E_{u}\right)$ and (c) helical $\left(A_{1 u}\right)$ pairings.
TABLE VI. $\Phi$ dependence and period of the maximum Josephson current $I_{c}$ in a symmetric SQUID.

\begin{tabular}{lcc}
\hline \hline Type of pairing & $\Phi$ dependence & Period \\
\hline (a) Chiral & symmetric & $\Phi_{0}$ \\
(b) Helical & symmetric & $\Phi_{0}$ \\
\hline \hline
\end{tabular}

Next, we discuss the helical $p$-wave case, where $I_{1}^{s}$ is given by

$$
I_{1}^{s}=\alpha \lambda+O\left(\lambda^{2}\right)
$$

This is because $I_{1}^{c}$ does not exist in the single-band model owing to the TRS of the helical $p$-wave pairing. Since $t_{5}$ only gives the effective phase shift of the pair potential, $I_{1}^{S}$ cannot be produced by $\lambda_{R}$. On the other hand, $\lambda$ directly induces $I_{1}^{S}$ in a similar manner as in the case of the chiral $p$-wave pairing.
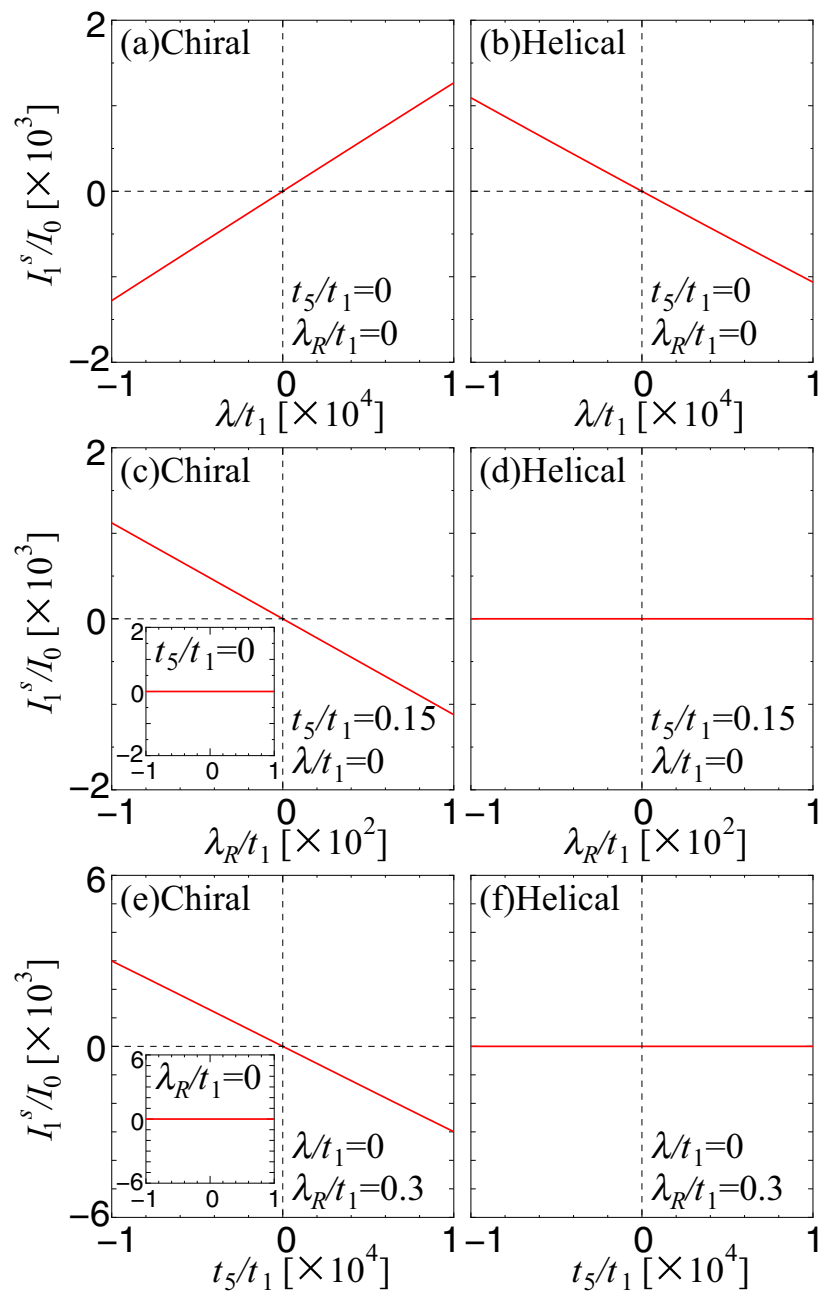

FIG. 10. $I_{1}^{s}$ [the coefficient of $\sin (\varphi)$ in the Fourier series of the current-phase relation in the junction] is plotted as a function of $\lambda$ (a), (b), $\lambda_{R}$ (c), (d), and $t_{5}$ (e), (f). Chiral pairing applies in (a), (c), and (e), and helical pairing with $\mathrm{A}_{1 u}$ symmetry in (b), (d), and (f). $t_{5}=0$ and $\lambda_{R}=0$ in (a) and (b). $t_{5} / t_{1}=0.15$ and $\lambda=0$ in (c) and (d). $\lambda=0$ and $\lambda / t_{1}=0.3$ in (e) and (f). 
We explain the physical origin of the term proportional to $\lambda$ in Eqs. (38) and (39) on the basis of the following arguments. Since calculation of the Josephson current in multiorbital system is a complicated problem, let us concentrate on the first term proportional to $\lambda$ in Eqs. (38) and (39). For this purpose, it is useful to focus on the symmetry of pair amplitude of Cooper pair. The first-order Josephson coupling term proportional to $\sin \varphi$ is nonzero only when the same symmetry of pair amplitude exists both in the left side $\mathrm{Sr}_{2} \mathrm{RuO}_{4}$ and the right side $s$-wave superconductors. It is known that for a singleorbital superconductor, the symmetry of Cooper pairs can be classified into four cases: (i) even-frequency spin-singlet even-parity (ESE) state, (ii) even-frequency spin-triplet oddparity (ETO) state, (iii) odd-frequency spin-triplet even-parity (OTE) state, and (iv) odd-frequency spin-singlet odd-parity (OSO) state $[25,28,74,75]$. In our model, there is only one orbital in the considered spin-singlet $s$-wave superconductor, which is in an even-frequency spin-singlet even-parity (ESE) state. On the other hand, we consider three orbitals in the left $\mathrm{Sr}_{2} \mathrm{RuO}_{4}$ superconducting electrode. With account of an orbital degree of freedom, Cooper pairs can be classified into eight classes: (i) even-frequency spin-singlet even-parity evenorbital (ESEE), (ii) even-frequency spin-triplet odd-parity even-orbital (ETOE), (iii) odd-frequency spin-triplet evenparity even-orbital (OTEE), (iv) odd-frequency spin-singlet odd-parity even-orbital (OSOE), (v) even-frequency spinsinglet odd-parity odd-orbital (ESOO), (vi) even-frequency spin-triplet even-parity odd-orbital (ETEO), (vii) oddfrequency spin-triplet odd-parity odd-orbital (OTOO), (viii) odd-frequency spin-singlet even-parity odd-orbital (OSEO) $[76,77]$. Due to the absence of orbital degree of freedom, the single orbital superconductor can be regarded as even parity with respect to exchange of orbitals. Thus, single orbital spin-singlet $s$-wave pairing belongs to the ESEE class. The symmetry breaking term in Hamiltonian converts original pairing symmetry into the other one. A bulk spin-orbit coupling term proportional to $\lambda$ can mix spin-singlet and spin-triplet states with simultaneous change of an orbital parity. Translational symmetry breaking mixes even and odd-frequency pairing by changing spatial parity. Orbital hybridization also mixes even and odd-frequency pairing by changing orbital parity $[76,77]$. By using these properties, we can discuss the origin of $\lambda$ term in Eqs. (38) and (39). Symmetry of pair potential of $\mathrm{Sr}_{2} \mathrm{RuO}_{4}$ is classified as ETOE. Due to the presence of the bulk spin-orbit coupling the $\lambda$ ESOO pairing state is generated, which in turn gets an admixture of OSEO state near the interface due to the translational symmetry breaking. Finally, OSEO pairing can generate the ESEE one at the interface, since three orbitals in $\mathrm{Sr}_{2} \mathrm{RuO}_{4}$ are not equivalent and the symmetry of orbital space in $\mathrm{Sr}_{2} \mathrm{RuO}_{4}$ is broken. In other words, spin-orbit coupling, orbital inequivalence, and broken translational symmetry are needed to generate ESEE pairing. The resulting ESEE state in $\mathrm{Sr}_{2} \mathrm{RuO}_{4}$ on the left side of the interface can couple to the ESEE state in an $s$-wave superconductor on the right side of the interface. This is the origin of the appearance of the first-order Josephson coupling term proportional to $\lambda$ in Eqs. (38) and (39).
In summary, we have studied Josephson currents in $\mathrm{SRO} / \mathrm{NM} / s$-wave junctions. We found that the first-order Josephson coupling is induced by the spin-orbit interaction for the cases of both chiral and helical $p$-wave pairings. Note that the $\sin (\varphi)$ term, which is absent in the single-band model, appears as a result of the spin-orbit interaction and interband hopping. In the case of helical pairing, the first-order Josephson term appears only in the three-band model. Owing to the existence of the first-order Josephson coupling, the period of the Josephson current, as the magnetic flux $\Phi$ is varied, is expected to become the period of the conventional junctions. For the case of chiral $p$-wave pairing, the Josephson current shows asymmetric behavior in the corner junction and the corner SQUID, owing to broken TRS. This asymmetry is enhanced by the spin-orbit interaction in the bulk SRO or at the interface in the junction. Since the magnitude of the spin-orbit interaction in SRO is not very small, it is possible to detect the asymmetry experimentally if the TRS breaking by chiral pairing is realized.

Recently, there has been a theoretical proposal about the momentum dependence of a chiral $p$-wave Cooper pair made from relatively distant sites, where the pair potentials given by $\sin 3 k_{x}+i \sin 3 k_{y}$ or $\sin k_{x} \cos k_{y}+i \cos k_{x} \sin k_{y}$ are considered [78]. This Cooper pair is thought to be hopeful for understanding the disappearance of the edge current. Since these pairings belong to the same irreducible representation of pair potential given by $\sin k_{x}+i \sin k_{y}$ where a Cooper pair is formed between nearest neighbor sites, it is natural to consider that the current phase relation of Josephson current between $s$-wave superconductors does not change qualitatively. Thus, the magnetic-field dependence of a corner Josephson junction satisfies $I(\Phi) \neq I(-\Phi)$.

In this paper, we assumed ballistic junctions with flat interfaces. Surface roughness and impurity scattering are known to influence the charge transport in spin-triplet $p$-wave superconductor junctions $[79,80]$ due to the anomalous proximity effect with enhanced zero energy local density of states driven by the induced odd-frequency spin-triplet $s$-wave pairing generated near the interface $[21,22]$. Then, the resulting Josephson current displays a low-temperature anomaly [21-23]. Taking into account the impurity-scattering effect in the multiband model is an interesting prospect for future work.

\section{ACKNOWLEDGMENTS}

This work was supported by a Grant-in-Aid for Scientific Research on Innovative Areas, Topological Material Science (Grants No. JP15H05851, No. JP15H05852, No. JP15H05853, and No. JP15K21717), a Grant-in-Aid for Scientific Research B (Grant No. JP15H03686), a Grant-in-Aid for Challenging Exploratory Research (Grant No. JP15K13498) from the Ministry of Education, Culture, Sports, Science, and Technology, Japan (MEXT); Japan-RFBR JSPS Bilateral Joint Research Projects/Seminars (Grants No. 15-52-50054 and No. 15668956); JSPS Core-to-Core Program (A. Advanced Research Networks); Dutch FOM; the Ministry of Education and Science of the Russian Federation, Grant No. 14.Y26.31.0007; and by the Russian Science Foundation, Grant No. 15-12-30030. 
[1] Y. Maeno, H. Hashimoto, K. Yoshida, S. Nishizaki, T. Fujita, J. G. Bednorz, and F. Lichtenberg, Nature (London) 372, 532 (1994).

[2] K. Ishida, H. Mukuda, Y. Kitaoka, K. Asayama, Z. Q. Mao, Y. Mori, and Y. Maeno, Nature (London) 396, 658 (1998).

[3] H. Murakawa, K. Ishida, K. Kitagawa, Z. Q. Mao, and Y. Maeno, Phys. Rev. Lett. 93, 167004 (2004).

[4] A. P. Mackenzie and Y. Maeno, Rev. Mod. Phys. 75, 657 (2003).

[5] Y. Maeno, S. Kittaka, T. Nomura, S. Yonezawa, and K. Ishida, J. Phys. Soc. Jpn. 81, 011009 (2012).

[6] T. M. Rice and M. Sigrist, J. Phys.: Condens. Matter 7, L643 (1995).

[7] K. Miyake and O. Narikiyo, Phys. Rev. Lett. 83, 1423 (1999).

[8] T. Kuwabara and M. Ogata, Phys. Rev. Lett. 85, 4586 (2000).

[9] T. Nomura and K. Yamada, J. Phys. Soc. Jpn. 69, 3678 (2000).

[10] T. Nomura and K. Yamada, J. Phys. Soc. Jpn. 71, 404 (2002).

[11] T. Nomura and K. Yamada, J. Phys. Soc. Jpn. 72, 2403 (2003).

[12] R. Arita, S. Onari, K. Kuroki, and H. Aoki, Phys. Rev. Lett. 92, 247006 (2004).

[13] T. Nomura, J. Phys. Soc. Jpn. 74, 1818 (2005).

[14] T. Nomura, D. S. Hirashima, and K. Yamada, J. Phys. Soc. Jpn. 77, 024701 (2008).

[15] Y. Yanase and M. Ogata, J. Phys. Soc. Jpn. 72, 673 (2003).

[16] S. Raghu, A. Kapitulnik, and S. A. Kivelson, Phys. Rev. Lett. 105, 136401 (2010).

[17] M. Sato and M. Kohmoto, J. Phys. Soc. Jpn. 69, 3505 (2000).

[18] K. Kuroki, M. Ogata, R. Arita, and H. Aoki, Phys. Rev. B 63, 060506 (2001).

[19] T. Takimoto, Phys. Rev. B 62, R14641 (2000).

[20] M. Tsuchiizu, Y. Yamakawa, S. Onari, Y. Ohno, and H. Kontani, Phys. Rev. B 91, 155103 (2015).

[21] Y. Tanaka and S. Kashiwaya, Phys. Rev. B 70, 012507 (2004).

[22] Y. Tanaka, S. Kashiwaya, and T. Yokoyama, Phys. Rev. B 71, 094513 (2005).

[23] Y. Asano, Y. Tanaka, and S. Kashiwaya, Phys. Rev. Lett. 96, 097007 (2006).

[24] Y. Tanaka, Y. Asano, A. A. Golubov, and S. Kashiwaya, Phys. Rev. B 72, 140503(R) (2005).

[25] Y. Tanaka and A. A. Golubov, Phys. Rev. Lett. 98, 037003 (2007).

[26] A. P. Schnyder, S. Ryu, A. Furusaki, and A. W. W. Ludwig, Phys. Rev. B 78, 195125 (2008).

[27] X.-L. Qi and S.-C. Zhang, Rev. Mod. Phys. 83, 1057 (2011).

[28] Y. Tanaka, M. Sato, and N. Nagaosa, J. Phys. Soc. Jpn. 81, 011013 (2012).

[29] J. Alicea, Rep. Prog. Phys. 75, 076501 (2012).

[30] Y. Ueno, A. Yamakage, Y. Tanaka, and M. Sato, Phys. Rev. Lett. 111, 087002 (2013).

[31] S. Kashiwaya, H. Kashiwaya, H. Kambara, T. Furuta, H. Yaguchi, Y. Tanaka, and Y. Maeno, Phys. Rev. Lett. 107, 077003 (2011).

[32] K. D. Nelson, Z. Q. Mao, Y. Maeno, and Y. Liu, Science 306, 1151 (2004).

[33] F. Laube, G. Goll, H. v. Löhneysen, M. Fogelström, and F. Lichtenberg, Phys. Rev. Lett. 84, 1595 (2000).

[34] H. Wang, W. Lou, J. Luo, J. Wei, Y. Liu, J. E. Ortmann, and Z. Q. Mao, Phys. Rev. B 91, 184514 (2015).

[35] A. Furusaki, M. Matsumoto, and M. Sigrist, Phys. Rev. B 64, 054514 (2001).
[36] K. Sengupta, H.-J. Kwon, and V. M. Yakovenko, Phys. Rev. B 65, 104504 (2002).

[37] M. Yamashiro, Y. Tanaka, and S. Kashiwaya, Phys. Rev. B 56, 7847 (1997).

[38] M. Yamashiro, Y. Tanaka, Y. Tanuma, and S. Kashiwaya, J. Phys. Soc. Jpn. 67, 3224 (1998).

[39] C. Honerkamp and M. Sigrist, J. Low Temp. Phys. 111, 895 (1998).

[40] Y. Tanaka and S. Kashiwaya, Phys. Rev. Lett. 74, 3451 (1995).

[41] S. Kashiwaya and Y. Tanaka, Rep. Prog. Phys. 63, 1641 (2000).

[42] C. R. Hu, Phys. Rev. Lett. 72, 1526 (1994).

[43] Y. Imai, K. Wakabayashi, and M. Sigrist, Phys. Rev. B 85, 174532 (2012).

[44] Y. Imai, K. Wakabayashi, and M. Sigrist, Phys. Rev. B 88, 144503 (2013).

[45] K. Yada, A. A. Golubov, Y. Tanaka, and S. Kashiwaya, J. Phys. Soc. Jpn. 83, 074706 (2014).

[46] F. Kidwingira, J. D. Strand, D. J. Harlingen, and Y. Maeno, Science 314, 1267 (2006).

[47] H. Kambara, S. Kashiwaya, H. Yaguchi, Y. Asano, Y. Tanaka, and Y. Maeno, Phys. Rev. Lett. 101, 267003 (2008).

[48] H. Kambara, T. Matsumoto, H. Kashiwaya, S. Kashiwaya, H. Yaguchi, Y. Asano, Y. Tanaka, and Y. Maeno, J. Phys. Soc. Jpn. 79, 074708 (2010).

[49] M. S. Anwar, T. Nakamura, S. Yonezawa, M. Yakabe, R. Ishiguro, H. Takayanagi, and Y. Maeno, Sci. Rep. 3, 2480 (2013).

[50] K. Saitoh, S. Kashiwaya, H. Kashiwaya, Y. Mawatari, Y. Asano, Y. Tanaka, and Y. Maeno, Phys. Rev. B 92, 100504 (2015).

[51] X.-L. Qi, T. L. Hughes, S. Raghu, and S.-C. Zhang, Phys. Rev. Lett. 102, 187001 (2009).

[52] M. Matsumoto and M. Sigrist, J. Phys. Soc. Jpn. 68, 3120 (1999).

[53] S.-I. Suzuki and Y. Asano, Phys. Rev. B 94, 155302 (2016).

[54] G. M. Luke, Y. Fudamoto, K. M. Kojima, M. I. Larkin, J. Merrin, B. Nachumi, Y. J. Uemura, Y. Maeno, Z. Q. Mao, Y. Mori, H. Nakamura, and M. Sigrist, Nature (London) 394, 558 (1998).

[55] J. Xia, Y. Maeno, P. T. Beyersdorf, M. M. Fejer, and A. Kapitulnik, Phys. Rev. Lett. 97, 167002 (2006).

[56] J. R. Kirtley, C. Kallin, C. W. Hicks, E.-A. Kim, Y. Liu, K. A. Moler, Y. Maeno, and K. D. Nelson, Phys. Rev. B 76, 014526 (2007).

[57] C. W. Hicks, J. R. Kirtley, T. M. Lippman, N. C. Koshnick, M. E. Huber, Y. Maeno, W. M. Yuhasz, M. B. Maple, and K. A. Moler, Phys. Rev. B 81, 214501 (2010).

[58] W. Huang, E. Taylor, and C. Kallin, Phys. Rev. B 90, 224519 (2014).

[59] S. Lederer, W. Huang, E. Taylor, S. Raghu, and C. Kallin, Phys. Rev. B 90, 134521 (2014).

[60] P. E. C. Ashby and C. Kallin, Phys. Rev. B 79, 224509 (2009).

[61] Y. Tada, N. Kawakami, and S. Fujimoto, New J. Phys. 11, 055070 (2009).

[62] T. Nomura and K. Yamada, J. Phys. Soc. Jpn. 71, 1993 (2002).

[63] Y. Asano, Y. Tanaka, M. Sigrist, and S. Kashiwaya, Phys. Rev. B 67, 184505 (2003).

[64] T. Oguchi, Phys. Rev. B 51, 1385 (1995).

[65] D. J. Singh, Phys. Rev. B 52, 1358 (1995).

[66] C. Noce and M. Cuoco, Phys. Rev. B 59, 2659 (1999).

[67] M. W. Haverkort, I. S. Elfimov, L. H. Tjeng, G. A. Sawatzky, and A. Damascelli, Phys. Rev. Lett. 101, 026406 (2008).

[68] A. Furusaki and M. Tsukada, Solid State Commun. 78, 299 (1991). 
[69] Y. Tanaka and S. Kashiwaya, Phys. Rev. B 56, 892 (1997).

[70] A. Umerski, Phys. Rev. B 55, 5266 (1997).

[71] D. J. Van Harlingen, Rev. Mod. Phys. 67, 515 (1995).

[72] Y. Asano, Y. Tanaka, M. Sigrist, and S. Kashiwaya, Phys. Rev. B 71, 214501 (2005).

[73] V. B. Geshkenbein, A. I. Larkin, and A. Barone, Phys. Rev. B 36, 235 (1987).

[74] Y. Tanaka, A. A. Golubov, S. Kashiwaya, and M. Ueda, Phys. Rev. Lett. 99, 037005 (2007).
[75] Y. Tanaka, Y. Tanuma, and A. A. Golubov, Phys. Rev. B 76, 054522 (2007).

[76] A. M. Black-Schaffer and A. V. Balatsky, Phys. Rev. B 88, 104514 (2013).

[77] Y. Asano and A. Sasaki, Phys. Rev. B 92, 224508 (2015).

[78] T. Scaffidi and S. H. Simon, Phys. Rev. Lett. 115, 087003 (2015).

[79] S. V. Bakurskiy, A. A. Golubov, M. Y. Kupriyanov, K. Yada, and Y. Tanaka, Phys. Rev. B 90, 064513 (2014).

[80] B. Lu, P. Burset, Y. Tanuma, A. A. Golubov, Y. Asano, and Y. Tanaka, Phys. Rev. B 94, 014504 (2016). 